LAPTH-1178/07

\title{
Hawking radiation of linear dilaton black holes
}

\author{
G. Clément ${ }^{a *}$ J.C. Fabris ${ }^{b \dagger}$ and G.T. Marques ${ }^{a, b \ddagger}$ \\ ${ }^{a}$ Laboratoire de Physique Théorique LAPTH (CNRS), \\ B.P.110, F-74941 Annecy-le-Vieux cedex, France \\ ${ }^{b}$ Departamento de Física, Universidade Federal do Espírito Santo, \\ Vitória, 29060-900, Espírito Santo, Brazil
}

April 3, 2007

\begin{abstract}
We compute exactly the semi-classical radiation spectrum for a class of non-asymptotically flat charged dilaton black holes, the socalled linear dilaton black holes. In the high frequency regime, the temperature for these black holes generically agrees with the surface gravity result. In the special case where the black hole is massless, we show that, although the surface gravity remains finite, there is no radiation, in agreement with the fact that massless objects cannot radiate.
\end{abstract}

\footnotetext{
*e-mail: gclement@lapp.in2p3.fr

${ }^{\dagger}$ e-mail: fabris@cce.ufes.br

${ }^{\ddagger}$ e-mail:gtadaiesky@cce.ufes.br
} 
Quantum field theory in curved spacetime predicts new phenomena such as particle emission by a black hole [1]. This is due to the fact that the vacuum for a quantum field near the horizon is different from the observer's vacuum at spatial infinity. A distant observer thus receives from a black hole a steady flux of particles exhibiting, in the high frequency regime, a black body spectrum with a temperature proportional to the surface gravity [2]. Although Hawking's original derivation of this black hole evaporation dealt with realistic collapsing black holes, Unruh [3] showed that the same results are obtained when the collapse is replaced by appropriate boundary conditions on the horizon of an eternal black hole. In the semi-classical approximation, the black hole radiation spectrum may be evaluated by computing the Bogoliubov coefficients relating the two vacua. An equivalent procedure is to compute the reflection and absorption coefficients of a wave by the black hole. Usually, the wave equation cannot be solved exactly, and one must resort to match solutions in an overlap region between the near-horizon and asymptotic regions [4, 5]. In the special case of the $(2+1)$ dimensional BTZ black hole [6], an exact solution of the wave equation is available, which allows for an exact computation of the radiation spectrum, leading to the Hawking temperature [7, 8, 9].

In this Letter, we discuss another case of black holes also allowing for an exact semi-classical computation of their radiation spectrum, that of linear dilaton black hole solutions to Einstein-Maxwell dilaton (EMD) theory in four dimensions. Linear dilaton black holes are a special case of the more general class of non-asymptotically flat black hole solutions to EMD [10, 11], which we first briefly present. We discuss the evaporation of these non-asymptotically flat black holes and show that they either collapse to a naked singularity in a finite time, or evaporate in an infinite time. We then specialize to linear dilaton black holes, and outline the analytical computation of their radiation spectrum. For massive black holes, this computation leads, in the high frequency regime, to the same temperature which is obtained from the surface gravity. However in the case of massless extreme black holes, we find that, although the surface gravity remains finite, there is no radiation, in agreement with the fact that a massless object cannot radiate.

EMD is defined by the following action

$$
S=\frac{1}{16 \pi} \int d x^{4} \sqrt{-g}\left[R-2 \partial_{\mu} \phi \partial^{\mu} \phi-e^{-2 \alpha \phi} F_{\mu \nu} F^{\mu \nu}\right],
$$

where $F_{\mu \nu}$ is the electromagnetic field, and $\phi$ is the dilatonic field, with coupling constant $\alpha$. This theory admits static spherically symmetric solutions 
representing black holes. Among these black hole solutions there are asymptotically flat ones [12, 13] as well as non-asymptotically flat configurations [10, 11]. In the present work, we are interested in the non-asymptotically flat black hole solutions

$$
\begin{aligned}
d s^{2} & =\frac{r^{\gamma}(r-b)}{r_{0}^{\gamma+1}} d t^{2}-\frac{r_{0}^{\gamma+1}}{r^{\gamma}(r-b)}\left\{d r^{2}+r(r-b) d \Omega^{2}\right\} \\
F & =\sqrt{\frac{1+\gamma}{2}} \frac{\nu}{r_{0}} d r \wedge d t, \quad e^{2 \alpha \phi}=\nu^{2}\left(\frac{r}{r_{0}}\right)^{1-\gamma} .
\end{aligned}
$$

with

$$
\gamma=\frac{1-\alpha^{2}}{1+\alpha^{2}}
$$

The constants $b$ and $r_{0}$ are related to the mass and to the electric charge of the black hole through

$$
M=(1-\gamma) b / 4, \quad Q=\sqrt{\frac{1+\gamma}{2}} \frac{r_{0}}{\nu} .
$$

The solutions (2),(3) interpolate between the Schwarzschild solution for $\gamma=-1\left(\alpha^{2} \rightarrow \infty\right)$ and the Bertotti-Robinson solution for $\gamma=+1\left(\alpha^{2}=0\right)$. For $b>0$ the horizon at $r=b$ hides the singularity at $r=0$, while in the extreme black hole case $b=0$ the horizon coincides with the singularity. This is a curious case, with vanishing mass but a finite electric charge. For $-1<\gamma<0\left(\alpha^{2}>1\right)$ the central singularity is timelike and clearly naked [11. On the other hand, for $0 \leq \gamma<1\left(0<\alpha^{2} \leq 1\right)$, the central singularity is null and marginally trapped [14], so that signals coming from the centre never reach external observers. Thus in this case, extreme black holes can be still considered as black holes indeed.

The statistical Hawking temperature of the black holes (2), computed as usual by dividing the surface gravity by $2 \pi$ is given by

$$
T_{H}=\frac{1}{4 \pi} \frac{b^{\gamma}}{r_{0}^{1+\gamma}} .
$$

It is finite for all $\gamma$ if $b \neq 0$. For $b=0$ and $-1<\gamma<0$ (naked singularity). the temperature is infinite, while for $b=0$ and $0<\gamma<1$ (extreme black hole), the temperature vanishes.

The case $b=\gamma=0$ is intriguing. Although this an extreme black hole, the situation is different from that of asymptotically flat extreme black holes. The near-horizon Euclidean extreme Reissner-Nordström geometry 
is cylindrical, rather than conical, so that its statistical temperature is arbitrary, contrary to the zero value derived from surface gravity [15]. In the present case the two-dimensional Euclidean continuation of the metric (2) with $\gamma=0$ clearly has a conical singularity at $r=b$ for all values of $b$, including $b=0$, leading for this particular extreme black hole to the finite temperature $T_{H}=1 / 4 \pi r_{0}$, in agreement with the value (6). However this result is questionable. A black hole with pointlike horizon and zero mass clearly cannot radiate, so one should rather expect its temperature to be zero. We will return to this question presently.

As black holes (2) radiate, they loose mass according to Stefan's law

$$
\frac{d M}{d t}=-\sigma A_{h} T_{H}^{4}
$$

where $\sigma$ is Stefan's constant, and $A_{h}=4 \pi r_{0}^{1+\gamma} b^{1-\gamma}$ is the horizon area. Assuming that only electrically neutral quanta are radiated, (7) implies that the horizon area decreases according to

$$
\frac{d b}{d t}=-\frac{4 \sigma}{(4 \pi)^{3}(1-\gamma)} r_{0}^{-3(1+\gamma)} b^{1+3 \gamma}
$$

which is solved by

$$
\begin{aligned}
b(t)=r_{0}\left(\frac{\gamma c}{1-\gamma} \frac{t-t_{0}}{r_{0}^{3}}\right)^{-1 / 3 \gamma} \quad(\gamma \neq 0), \\
b(t)=r_{0} \exp \left(-\frac{c}{3} \frac{t-t_{0}}{r_{0}^{3}}\right) \quad(\gamma=0),
\end{aligned}
$$

where $c=3 \sigma / 16 \pi^{3}$, and $t_{0}$ is an integration constant. The outcome depends on the sign of $\gamma$. For $\gamma<0$, the Hawking temperature increases with decreasing mass and the black hole collapses to a naked singularity (or evaporates away altogether in the Schwarzschild case $\gamma=-1$ ) in a finite time according to $b \sim\left(t_{0}-t\right)^{1 / 3|\gamma|}$. On the other hand, for $\gamma \geq 0$, the Hawking temperature decreases (or is constant for $\gamma=0$ ) with decreasing mass, and the black hole evaporates in an infinite time, reaching the extreme black hole state $b=0$ only asymptotically.

We now proceed to a more precise evaluation of the temperature of nonasymptotically flat black holes from the study of wave scattering in these spacetimes. The wave equation

$$
\nabla^{2} \phi=0
$$


does not generically allow for an exact solution in the spacetimes (2). However, it can be solved analytically [16] in the case of linear dilaton black holes with $\gamma=0$ and $b \neq 0$, with the metric

$$
d s^{2}=\frac{r-b}{r_{0}} d t^{2}-\frac{r_{0}}{r-b}\left\{d r^{2}+r(r-b) d \Omega^{2}\right\},
$$

Considering the harmonic eigenmodes

$$
\phi(x)=\psi(r, t) Y_{l m}(\theta, \varphi), \quad \psi(r, t)=R(r) \mathrm{e}^{-i \omega t},
$$

we obtain the following radial equation:

$$
\partial_{r}\left(r(r-b) \partial_{r} R\right)+\left(\bar{\omega}^{2} \frac{r}{r-b}-l(l+1)\right) R=0
$$

$\left(\bar{\omega}^{2} \equiv \omega^{2} r_{0}^{2}\right)$. Putting

$$
y=\frac{b-r}{b}, \quad R=y^{i \bar{\omega}} f,
$$

reduces (13) to the equation

$$
y(1-y) \partial_{y}^{2} f+(1+2 i \bar{\omega}-2(1+i \bar{\omega}) y) \partial_{y} f+\left(\bar{\omega}^{2}-i \bar{\omega}-\bar{\lambda}^{2}-1 / 4\right) f=0,
$$

with

$$
\bar{\lambda}^{2}=\bar{\omega}^{2}-(l+1 / 2)^{2} .
$$

This is a hypergeometric equation

$$
y(1-y) \partial_{y}^{2} f+(c-(a+b+1) y) \partial_{y} f-a b f=0,
$$

with

$$
a=\frac{1}{2}+i(\bar{\omega}+\bar{\lambda}), \quad b=\frac{1}{2}+i(\bar{\omega}-\bar{\lambda}), \quad c=1+2 i \bar{\omega} .
$$

It follows that the general solution of equation (13) is

$$
\begin{aligned}
& R=C_{1}\left(\frac{r-b}{b}\right)^{i \bar{\omega}} F\left(\frac{1}{2}+i(\bar{\omega}+\bar{\lambda}), \frac{1}{2}+i(\bar{\omega}-\bar{\lambda}), 1+2 i \bar{\omega} ; \frac{b-r}{b}\right) \\
& +\quad C_{2}\left(\frac{r-b}{b}\right)^{-i \bar{\omega}} F\left(\frac{1}{2}-i(\bar{\omega}+\bar{\lambda}), \frac{1}{2}-i(\bar{\omega}-\bar{\lambda}), 1-2 i \bar{\omega} ; \frac{b-r}{b}\right) .
\end{aligned}
$$

Putting

$$
\frac{r-b}{b}=\mathrm{e}^{x / r_{0}}
$$


the partial wave near the horizon $(x \rightarrow-\infty)$ is thus

$$
\psi \simeq C_{1} \mathrm{e}^{i \omega(x-t)}+C_{2} \mathrm{e}^{-i \omega(x+t)} .
$$

To obtain the behavior of the partial wave near spatial infinity, we must expand the solutions of (15) in hypergeometric functions of argument $1 / y$. The relevant transformation is

$$
\begin{aligned}
F(a, b, c ; y) & =\frac{\Gamma(c) \Gamma(b-a)}{\Gamma(b) \Gamma(c-a)}(-y)^{-a} F(a, a+1-c, a+1-b ; 1 / y) \\
& +\frac{\Gamma(c) \Gamma(a-b)}{\Gamma(a) \Gamma(c-b)}(-y)^{-b} F(b, b+1-c, b+1-a ; 1 / y)
\end{aligned}
$$

This leads to the asymptotic behavior

$$
\psi \simeq\left(\frac{r}{b}\right)^{-1 / 2}\left(B_{1} \mathrm{e}^{i(\lambda x-\omega t)}+B_{2} \mathrm{e}^{-i(\lambda x+\omega t)}\right)
$$

$\left(\lambda=\bar{\lambda} / r_{0}\right)$, where the amplitudes of the asymptotic outgoing and ingoing waves $B_{1}$ and $B_{2}$ are related to the amplitudes of the near-horizon outgoing and ingoing waves $C_{1}$ and $C_{2}$ by

$$
\begin{aligned}
B_{1} & =\Gamma(2 i \bar{\lambda})\left[\frac{\Gamma(1+2 i \bar{\omega})}{\Gamma(1 / 2+i(\bar{\omega}+\bar{\lambda}))^{2}} C_{1}+\frac{\Gamma(1-2 i \bar{\omega})}{\Gamma(1 / 2-i(\bar{\omega}-\bar{\lambda}))^{2}} C_{2}\right] \\
B_{2} & =\Gamma(-2 i \bar{\lambda})\left[\frac{\Gamma(1+2 i \bar{\omega})}{\Gamma(1 / 2+i(\bar{\omega}-\bar{\lambda}))^{2}} C_{1}+\frac{\Gamma(1-2 i \bar{\omega})}{\Gamma(1 / 2-i(\bar{\omega}+\bar{\lambda}))^{2}} C_{2}\right] .
\end{aligned}
$$

Hawking radiation can be considered as the inverse process of scattering by the black hole, with the asymptotic boundary condition $B_{1}=0$ (the outgoing mode is absent). The coefficient for reflection by the black hole is then given by

$$
R=\left.\frac{\left|C_{1}\right|^{2}}{\left|C_{2}\right|^{2}}\right|_{B_{1}=0}=\frac{\left|\Gamma(1 / 2+i(\bar{\omega}+\bar{\lambda}))^{2}\right|^{2}}{\left|\Gamma(1 / 2+i(\bar{\omega}-\bar{\lambda}))^{2}\right|^{2}}=\frac{\cosh ^{2} \pi(\bar{\omega}-\bar{\lambda})}{\cosh ^{2} \pi(\bar{\omega}+\bar{\lambda})} .
$$

The resulting radiation spectrum is

$$
N=\frac{R}{1-R}=\left(\mathrm{e}^{\omega / T_{H}}-1\right)^{-1} .
$$

For high frequencies, $\bar{\lambda} \simeq \bar{\omega}=\omega / r_{0}$, and we recover from (25) the Hawking temperature as computed from the surface gravity,

$$
T_{H}=\frac{1}{4 \pi r_{0}} .
$$


The above computation fails in the linear dilaton vacuum case $b=0$. The question of assigning a temperature to such massless black holes might be evacuated by arguing that they cannot be formed, either through central collapse of matter, or (as we have seen above) through evaporation of massive black holes. Nevertheless, as a matter of principle one should consider the possibility of primordial massless black holes. From the general temperature law (6) these should have a finite temperature. On the other hand, being massless they cannot radiate energy away, so their temperature should vanish.

The question can be settled by solving the massless Klein-Gordon equation in the metric (11) with $b=0$,

$$
d s^{2}=\frac{r}{r_{0}} d t^{2}-\frac{r_{0}}{r} d r^{2}-r_{0} r d \Omega^{2} .
$$

This metric can be rewritten as

$$
d s^{2}=\Sigma^{2}\left[d \tau^{2}-d x^{2}-d \Omega^{2}\right]
$$

with

$$
x=\ln \left(r / r_{0}\right), \quad \tau=t / r_{0}, \quad \Sigma=r_{0} \mathrm{e}^{x / 2},
$$

showing that the linear dilaton vacuum metric is conformal to the product $M_{2} \times S_{2}$ of a two-dimensional Minkowski spacetime with the two-sphere. Performing also the redefinition

$$
\phi=\Sigma^{-1} \psi,
$$

the Klein-Gordon equation (10) is reduced to

$$
\nabla^{2} \phi=\Sigma^{-3}\left[\partial_{\tau \tau}-\partial_{x x}-\nabla_{\Omega}^{2}+\frac{1}{4}\right] \psi=0,
$$

where $\nabla_{\Omega}^{2}$ is the Laplacian operator on the two-sphere.

For a given spherical harmonic with orbital quantum number $l$, the reduced Klein-Gordon equation is thus

$$
\nabla_{2}^{2} \psi_{l}+(l+1 / 2)^{2} \psi_{l}=0,
$$

with $\nabla_{2}^{2}$ the Dalembertian operator on $M_{2}$. Also, for a given spherical harmonic the four-dimensional Klein-Gordon norm reduces to the $M_{2}$ norm:

$$
\|\phi\|^{2}=\frac{1}{2 i} \int d^{3} x \sqrt{|g|} g^{0 \mu} \phi^{*} \stackrel{\leftrightarrow}{\partial_{\mu}} \phi=\frac{2 \pi}{i} \int d x \psi_{l}^{*} \stackrel{\leftrightarrow}{\partial_{\tau}} \psi_{l}
$$


Thus, the problem of wave propagation in the linear dilaton vacuum reduces to the propagation of eigenmodes of a free Klein-Gordon field in two dimensions, with effective mass $\mu=l+1 / 2$. Clearly there is no reflection, so that the linear dilaton vacuum does not radiate and hence its Hawking temperature vanishes, contrary to the naive surface gravity value (6). A similar reasoning holds in $2+1$ dimensions for the BTZ vacuum [6] $(M=L=0)$, which is conformal to $M_{2} \times S_{1}$.

We have shown that a complete analytical computation of the radiation spectrum is possible for linear dilaton black hole solutions of EMD. For massive black holes, this leads in the high frequency regime to a Planckian distribution with a temperature independent of the black hole mass, in accordance with the surface gravity value. On the other hand, we find that extreme, massless black holes do not radiate, thereby solving the paradox presented by apparently hot (if the surface gravity temperature is taken seriously) yet massless black holes.

Acknowledgements: J.C.F. thanks the LAPTH for the warm hospitality during the elaboration of this work. He also thanks CNPq (Brazil) for partial support. J.C.F. and G.T.M. thank the French-Brazilian scientific cooperation CAPES/COFECUB for partial financial support.

\section{References}

[1] N.D. Birrell and P.C.W. Davies, Quantum fields in curved space, Cambridge University Press, Cambridge (1982).

[2] S.W. Hawking, Commun. Math. Phys. 43 (1975) 199.

[3] W.G. Unruh, Phys. Rev. D14 (1976) 870.

[4] D. Page, Phys. Rev. D13 (1976) 198.

[5] W.G. Unruh, Phys. Rev. D14 (1976) 3251.

[6] M. Bañados, C. Teitelboim and J. Zanelli, Phys. Rev. Lett. 69 (1992) 1849.

[7] K. Ghoroku and A.L. Larsen, Phys. Lett. B328 (1994) 28.

[8] M. Natsuume, N. Sakai and M. Sato, Mod. Phys. Lett. A11 (1996) 1467.

[9] D. Birmingham, I. Sachs and S. Sen, Phys. Lett. B413 (1997) 281. 
[10] K.C.K. Chan, J.H. Horne and R.B. Mann, Nucl. Phys. B447 (1995) 441.

[11] G. Clément and C. Leygnac, Phys. Rev. D70 (2004) 084018.

[12] G.W. Gibbons and K. Maeda, Nucl. Phys. B298 (1988) 741.

[13] D. Garfinkle, G.T. Horowitz and A. Strominger, Phys. Rev. D43 (1991) 3140 .

[14] S.A. Hayward, Class. Quantum Grav. 17 (2000) 4021.

[15] S.W. Hawking, G.T. Horowitz and S.F. Ross, Phys. Rev. D51 (1995) 4302 .

[16] G. Clément, D. Gal'tsov and C. Leygnac, Phys. Rev. D67 (2003) 024012 . 